stellar absorption affects the observed distributions. If, optically, one desires to list directions for which one is most likely looking along a spiral arm-then one can do so only on the basis of exhaustive regional surveys based on spectra, $U B V$ colours, absolute magnitude estimates, and radial velocities.

Westerlund: The possible supernova remnant at $14^{\mathrm{h}} 38^{\mathrm{m}},-62^{\circ} 30^{\prime}$ has been photographed in red light with the Uppsala telescope. The apparent centre of the brightest arc (RCW No. 86) is about $4^{\prime}$ from the peak-temperature $\left(4 \cdot 8^{\circ} \mathrm{K}\right)$ point of the radio source. Another weak arc follows exactly the northern $2^{\circ} \mathrm{K}$ contour. The inside of the first arc is rich in filaments and one filament goes through the $4 \cdot 8^{\circ} \mathrm{K}$ point. The length of the most pronounced arc is $\sim 5^{\prime}$. The structure of the filaments does not indicate an ordinary HII region. We hope to obtain a spectrum of the brightest filament with our nebular spectrograph.

\title{
27. OBSERVATIONS DE RESTES DE SUPERNOVAE A LA STATION DE RADIOASTRONOMIE DE NANÇAY
}

\section{A. Boischot et J. Lequeux \\ Observatoire de Paris-Meudon}

La structure de restes de supernovae galactiques de petit diamètre apparent a été étudiée sur $1420 \mathrm{Mc} / \mathrm{s}$ avec l'interféromètre à 2 antennes de Nançay, dont la résolution maximale est $18^{\prime \prime} \mathrm{EW}$ et $\mathrm{l}^{\prime} \mathrm{NS}$ (Lequeux 1962). Taurus A a des dimensions de $3^{\prime}, 9 \times 2^{\prime}, 7$ à demi-intensité, l'angle de position du grand axe étant $126^{\circ}$. L'émissivité dans cette radiosource croît rapidement vers le centre; d'autre part ses dimensions augmentent avec la longueur d'onde, ce qui peut s'expliquer d'après Woltjer (1958) par une création continue d'électrons relativistes au centre de la radiosource. Cassiopeia A, au contraire de Taurus A, est parfaitement circulaire; en admettant la symétrie sphérique, nos observations montrent que presque toute l'émission radioélectrique provient d'une enveloppe sphérique de $2^{\prime}, 0$ de rayon et $0^{\prime}, 6$ d'épaisseur à demi émissivité. Les résultats obtenus sur les Supernovae de Képler et Tycho Brahé sont en accord avec ceux de Cal Tech sur $960 \mathrm{Mc} / \mathrm{s}$ : la Supernova de Tycho Brahé est peut-être une enveloppe sphérique, mais on ne peut rien dire de celle de Képler.

D'autres restes de supernovae de plus grand diamètre ont été étudiés plus récemment avec la partie actuellement construite $(40 \mathrm{~m} \times 35 \mathrm{~m})$ du grand radiotélescope de Nançay, dont le pouvoir de résolution est environ $20^{\prime} \times 20^{\prime}$ à $1430 \mathrm{Mc} / \mathrm{s}$ et $12^{\prime} \times 12^{\prime}$ à $2300 \mathrm{Mc} / \mathrm{s}$. Une carte d'isophotes de HB 21 sur $1430 \mathrm{Mc} / \mathrm{s}$ (Boischot 1962) montre une structure en anneau que l'on retrouve également dans d'autres restes de supernovae (IC 443, HB 9, CTA 1), et dans la Nébuleuse de la Rosette, dont on peut se demander si elle n'est pas aussi un reste de supernova. Sur la carte de HB 21 publiée par Boischot (1962), la déclinaison est erronée et doit être augmentée de $30^{\prime}$. Enfin, W44 et la partie non-thermique de W28 ont été dédoublées en ascension droite sur $2300 \mathrm{Mc} / \mathrm{s}$ et sont sans doute des restes de supernovae en forme d'enveloppe sphérique; la source non-thermique dans W28, dont le diamètre est $28^{\prime}$ et l'indice spectral $-0,37 \pm 0,10$, est identifiée à un anneau de $6^{\prime}$ de diamètre, entouré de fragments d'anneaux concentriques s'étendant sur $24^{\prime}$ de diamètre, bien visibles sur le Sky Atlas (Nguyen Quang 1963). 
Ainsi la structure en enveloppe sphérique semble caractéristique des restes de supernovae, Taurus A constituant une exception remarquable. Néanmoins la théorie de van der Laan (1962) ne peutêtre appliquée qu'aux objets de très faible brillance radioélectrique. Dans le cas de Cassiopeia A, le champ magnétique et les électrons relativistes proviennent sans doute de l'explosion initiale. Une modification de la théorie de Shklovsky (1960) pour le cas d'une enveloppe sphérique en expansion conduit, en estimant à $6 \times 10^{-4}$ gauss la valeur actuelle du champ magnétique obtenue en supposant l'équipartition de l'énergie magnétique et de l'énergie des particules, à l'estimation de la masse de l'enveloppe, soit $2,3 M_{\odot}$, et de la décroissance annuelle du flux radioélectrique, soit 1,7\%, en accord raisonnable avec l'observation. Mais il paraît prématuré d'avancer une théorie générale des restes de supernovae de forte et de moyenne brillance. Cette théorie devra en particulier rendre compte des différences considérables qui existent entre l'indice spectral de ces radiosources.

\section{Références}

Boischot, A. (1962).-C.R. 255: 3374-6.

VAN DER LAAN, H. (1962).-M.N. 124 : 125-45.

LequeUX, J. (1962).-Ann. d'Ast. 25: 221-60.

Nguyen. Quang, R. (1963).--Notes et Informations (Observatoire de Paris) Fasc. XI: Radioastronomie No. 2.

ShkLovsky, I. S. (1960).-Soviet Astronomy-AJ 4: 243-9.

WOLTJER, L. (1958).-B.A.N. 14: 39-80.

\section{Discussion}

Bolton: I believe the development of supernova remnants may be considerably affected by their interstellar environment. For example the Crab nebula and Shajn 147, which have perfectly symmetrical forms, are in regions of relatively low interstellar density. On the other hand, Cas A, and Tycho's and Kepler's supernovae may be in regions of very high density. While filament velocities of $\sim 3000 \mathrm{~km} / \mathrm{sec}$ exist in Cas $\mathrm{A}$, some of the filaments are almost stationary.

Lequeux: In the case of Cas A, there is probably not much interaction with the interstellar medium with respect to radio waves if we put the age at 260 years.

Komesaroff: Cassiopeia A is similar to Puppis A, although Cas A is 10 to 20 times larger in angular diameter than Pup A. Both sources appear to be unpolarized.

Buscombe: Can one determine the age of supernova remnants from radio measurements?

Lequeux: No, only from proper motion studies.

Mathewson: There is a similarity between the appearance of this source (Rosette nebula) and the Cygnus loop.

Davies: The Cygnus loop has sharp edges compared with the Rosette nebula, where the intensity falls off gradually from the brightest part of the ring.

Hill: I thought that the Rosette nebula was a thermal source excited by a cluster of early-type stars.

Lequeux: Yes, radio and $\mathrm{H} \alpha$ brightnesses compare well for an HII region. 